\title{
Pensamento crítico e a formação de profissionais em Odontologia: uma revisão narrativa da literatura
}

Cynthia Moura Louzada Farias*, Raquel Baroni de Carvalho**, Lara Paulino Laiber***, Lorrayne Belotti****, Karina Tonini dos Santos Pacheco*****

\footnotetext{
Mestre em Clínica Odontológica; professora voluntária do Curso de Odontologia da UFES.

** Professora Associada do Departamento de Medicina Social da UFES.

*** Mestranda em Clínica Odontológica, UFES.

**** Mestranda em Saúde Coletiva, UFES.

****** Professora Adjunta do Departamento de Medicina Social da UFES.
}

\section{RESUMO}

O objetivo do estudo foi investigar e descrever, por meio de uma revisão narrativa da literatura, evidências do pensamento crítico no processo de formação de profissionais em saúde, dentre eles o cirurgião-dentista. Os dados foram coletados nas bases Medline, Lilacs e SciELO, selecionando textos completos na área das ciências da saúde, especificamente, Medicina, Enfermagem e Odontologia. A análise descritiva dos resultados revelou três categorias, sobre as quais conduziu-se o estudo. A análise final foi realizada com 25 artigos do total de 211 encontrados. Os resultados mostraram que $76,2 \%$ dos trabalhos foram publicados entre 2009 e 2015, apenas 33,3\% são brasileiros e $66,7 \%$ expressam análises e resultados de publicações internacionais. Verificou-se, também, que $66,7 \%$ dos estudos abordavam o pensamento crítico na Odontologia, dentre os quais $9,5 \%$ eram brasileiros. Conclui-se que são evidentes os benefícios que habilidades envolvendo o pensamento crítico podem proporcionar na formação de profissionais da saúde. O pensamento crítico possibilita que os profissionais tornem-se mais reflexivos e questionadores, requisitos essenciais à tomada de decisões num mundo tão complexo.

Descritores: Educação Baseada em Competências. Odontologia. Educação Superior. Pensamento. Revisão.

\section{INTRODUÇÃO}

Para começarmos a definir o que é pensamento crítico, é importante destacar que todos nós, seres humanos, pensamos. Mas, se todos nós pensamos, qual a diferença entre pensamento e pensamento crítico? A diferença é o controle do pensamento, pois "o pensamento é basicamente qualquer atividade mental e pode ser sem objetivo e descontrolado". Ele pode servir a um propósito, entretanto, não fomos despertados para os seus benefícios. A mente humana não permite a lembrança de todos os nossos pensamentos. A priori, “o pensamento crítico é controlado, proposital e mais apropriado para levar aos benefícios óbvios resultantes"1. 
Desenvolver a capacidade de observação significa estimular o ato reflexivo, que conduz ao pensamento crítico e tende a ampliar os horizontes de quem assim o faz, como um agente ativo das transformações da sociedade, ao buscar interagir com a realidade ${ }^{2}$.

$\mathrm{O}$ século XXI apresenta-se em um cenário de grande valorização do conhecimento e na respectiva capacidade de inovação promovida pelas pessoas. $\mathrm{Na}$ sociedade atual percebe-se elevada demanda por profissionais altamente qualificados. Essa crescente demanda por qualidade em todos os setores da sociedade reflete na vida dos cidadãos, tanto pessoal, quanto profissional. Existe a crescente dúvida sobre quais competências são mais importantes para que os indivíduos enfrentem os desafios do seu século. A resposta a esta pergunta demanda reflexão profunda sobre as habilidades e competências desenvolvidas e estimuladas ${ }^{3}$.

Longe de ser um modismo, o desenvolvimento de habilidades e competências sempre foi objetivo da educação e precisa ser entendido como um processo de formação integral, que não se restringe à transmissão de conteúdos. Em busca de atender às complexidades do cotidiano, a qualidade do conhecimento e as competências envolvidas tornaram-se imprescindíveis ao exercício profissional qualificado em decisões que exigem o pensamento crítico, a fim de identificar as necessidades do paciente e implementar as melhores práticas ${ }^{3,4}$.

Pensar criticamente é uma habilidade que deve ser usada em qualquer momento em que se esteja para decidir o quê, como ou porquê fazer. Vale a pena destacar que o pensamento crítico não é um método a ser aprendido, mas um processo, uma orientação da mente, incorporando os domínios afetivo e cognitivo, e essa habilidade deve ser bem desenvolvida e trabalhada no contexto acadêmico, estimulando a investigação e promovendo o raciocínio do futuro profissional $^{5}$.

A habilidade de pensamento crítico permite uma mudança de cultura, de compreensão de vida, sendo um elemento necessário para o sucesso profissional do acadêmico, principalmente na área da saúde 6 . Estudos comprovaram a relação entre práticas de ensino que estimulam o pensamento crítico e a alta performance de desempenho do futuro profissional. Isso porque a partir dele são estimulados a desenvolver competências, ou seja, atitudes para melhor desempenho nas atividades profissionais, tendo como principais componentes a organização e planejamento do trabalho, a inovação e capacidade para resolver situações não rotineiras, incluindo a capacidade necessária para relacionar-se com as mais diversas pessoas, além de mais habilidade na tomada de decisões ${ }^{7}$.

Assim, diante das necessidades de adequações e mudanças no processo de formação dos profissionais de saúde propostas pelas Diretrizes Curriculares Nacionais (DCN), justifica-se o presente estudo, que teve o objetivo investigar e descrever, por meio de uma revisão da literatura, evidências da importância do pensamento crítico no processo de formação e no cotidiano dos profissionais em saúde, onde estão inseridos os profissionais da Odontologia.

\section{MÉTODOS}

Trata-se de revisão da literatura que aborda trabalhos publicados sobre $\mathrm{o}$ pensamento crítico no processo de formação dos profissionais da saúde, entre eles o 
cirurgião-dentista. $\mathrm{O}$ material foi coletado nas bases eletrônicas de dados Medline, Lilacs e SciELO. Foram selecionados os periódicos com textos completos, na área das ciências da saúde, especificamente, Medicina, Enfermagem e Odontologia. Foram definidas estratégias de busca diferentes requeridas para cada base de dados, com os descritores: "critical thinking in health; critical thinking in dentistry; higher education; educação baseada em competências; odontologia; educação superior; educação em enfermagem; pensamento", usando-se para busca o modo avançado, uma ou mais palavras, "AND/ OR" para expressões, palavras no título, resumo (abstract) e ou no artigo, por meio do método integrado de busca.

Nesta fase, foram encontrados 229 artigos para os descritores selecionados. Como critérios de inclusão foram considerados artigos originais em inglês e ou português, revisados por pares, publicados no período de janeiro de 2004 a janeiro 2016.
Este último critério foi estabelecido pela disseminação do tema no meio acadêmico, nesse período. Após utlizado o ano de publicação como critério de exclusão/inclusão, reduziu-se para 85 o número de artigos. Na sequência, passou-se ao critério revisão por pares, restando 80 trabalhos. Após a leitura dos resumos, 41 artigos foram selecionados e lidos na íntegra e 25 incluídos para análise, por preencherem todos os critérios de inclusão pré-estabelecidos.

Por meio da análise descritiva dos dados, pôde-se estabelecer os assuntos para nortear a discussão, entre eles a importância do pensamento crítico no ensino superior, o pensamento crítico na saúde e o pensamento crítico na Odontologia.

\section{RESULTADOS}

Ao todo foram analisados 25 artigos. A frequência numérica dos trabalhos encontrados, selecionados e os incluídos na revisão podem ser verificados na tabela 1 .

Tabela 1- Resultado das estratégias de busca realizadas nas bases de dados selecionadas, segundo critérios de inclusão e exclusão, entre 2004-2016

\begin{tabular}{lccccc}
\hline & $\begin{array}{c}\text { Artigos } \\
\text { Encontrados }\end{array}$ & \multicolumn{2}{c}{ Artigos Excluídos } & Artigos \\
\cline { 3 - 5 } & & Duplicidade & Resumo & Íntegra & Incluídos \\
\hline Base de dados & 179 & 13 & 49 & 24 & 16 \\
Medline & 32 & 3 & 20 & 11 & 7 \\
SciELO & 18 & 5 & 11 & 6 & 2 \\
Lilacs & & & &
\end{tabular}

Os dados mostram que são escassas as publicações de estudos sobre o pensamento crítico na área da saúde, no cenário nacional, e mais ainda na Odontologia. Os artigos que abordavam o pensamento crítico em Medicina, Enfermagem e Odontologia foram categorizados em "pensamento crítico na saúde (PCS)", juntamente com os que tratavam exclusivamente a Medicina e ou a Enfermagem. Os artigos categorizados em "pensamento crítico na odontologia (PCO)" tratavam exclusivamente dessa área (figura 1). 


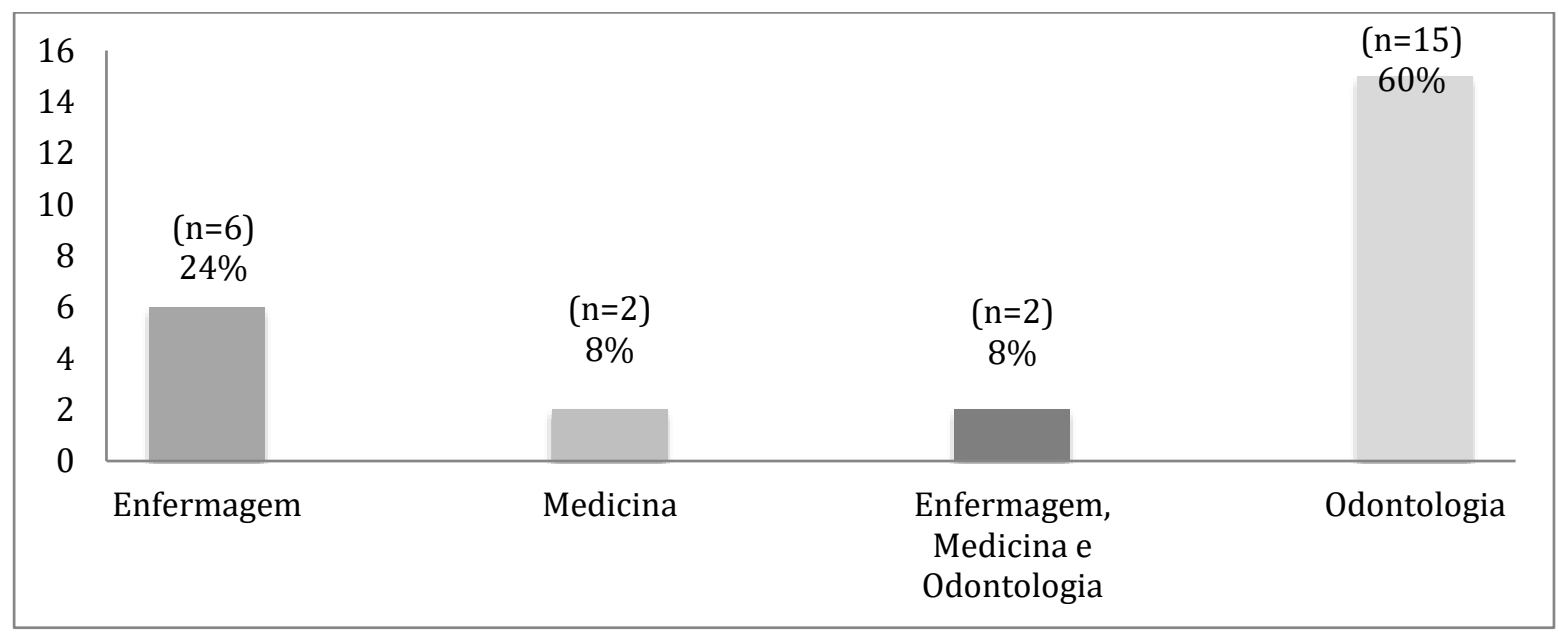

Figura 1. Frequência percentual dos artigos categorizados em "Pensamento Crítico na Saúde" e "Pensamento Crítico na Odontologia" - período de janeiro de 2004 a janeiro 2016

Das publicações analisadas, 76,2\% (16) foram publicadas entre 2009 e 2014. Dessas, apenas 33,3\% (7) são nacionais e $66,7 \%$ (14) são publicações internacionais. As publicações que mostram a habilidade do pensamento crítico na saúde corresponderam a $40 \%$ (10) e $60 \%$ (15) dos estudos abordavam o pensamento crítico na Odontologia, mas apenas $9,5 \%$ (2) eram publicações nacionais (tabela 2).

\section{DISCUSSÃO}

A discussão sobre o papel e a importância da competência do pensamento crítico na educação e na formação em saúde ganhou espaço no mundo inteiro ao longo das últimas décadas. Nos anos 80, representaram um importante passo para o desenvolvimento e a compreensão do ser humano em sua integralidade, permitindo desvelar a realidade e propor ações transformadoras.

Considerando-se as necessidades de mudanças requeridas na formação profissional na área da saúde ${ }^{8,9}$ com o desenvolvimento de habilidades e competências como parte no processo de formação integral dos acadêmicos, pretendidas pelas mudanças sociais e pelas
$\mathrm{DCN}^{10}$, observou-se na revisão sistemática realizada, que são poucas as pesquisas sobre os pressupostos do pensamento crítico na Odontologia no cenário brasileiro.

No Brasil, constantemente, tem-se levantado discussões a respeito da necessidade de mudanças no ensino em saúde, que precisa ser atualizado e adequado às finalidades relevantes requeridas pela população em cada momento específico ${ }^{11}$. Como regra geral, o estímulo ao pensamento crítico é apontado como uma variável fundamental para dar conta da complexidade das ações em saúde, as quais exigem, para obter êxito, que o profissional seja comprometido com a realidade concreta de sua prática ${ }^{12}$.

Desenvolver competências e habilidades sempre foi objetivo da educação, e pensar criticamente tem uma importância fundamental, diante da natureza dinâmica dos cuidados de saúde a garantia de ter desenvolvido a habilidade do pensamento crítico auxiliam no processo de construção da confiança em alcançar seu potencial. $\mathrm{O}$ pensamento crítico é uma competência que vem como uma das saídas para reconectar o indivíduo ao mundo onde vive, e melhor com- 
Tabela 2 - Descrição dos aspectos relevantes sobre o pensamento crítico encontrados nos artigos PCO e PCS incluídos na revisão - período entre janeiro de 2004 a janeiro 2016 (continua)

\begin{tabular}{|c|c|c|c|c|}
\hline Autor(es) & Ano & Periódico & Categoria & Abordagens Relevantes \\
\hline Fagundes, Burnham ${ }^{16}$ & 2005 & $\begin{array}{l}\text { Comunicação, } \\
\text { Saúde, } \\
\text { Educação }\end{array}$ & PCS & $\begin{array}{l}\text { Propuseram compartilhar experiências e saberes em espaços de aprendizagem no trabalho. } \\
\text { Afirmaram a necessidade de repensar as práticas universitárias para formarem os futuros } \\
\text { profissionais com habilidades técnicas, mais críticos em suas decisões. }\end{array}$ \\
\hline Pires, Bueno ${ }^{20}$ & 2009 & $\begin{array}{l}\text { Acta Paulista } \\
\text { Enfermagem }\end{array}$ & PCS & $\begin{array}{l}\text { Afirmaram que há necessidade de novas metodologias, e maior articulação entre modelos de } \\
\text { teorias e prática. Concluíram que a relação interdiscente e interdocente auxilia a construção do } \\
\text { pensamento crítico que se forma a partir do diálogo, e deve ser compartilhado diariamente nos } \\
\text { cursos de medicina, enfermagem e odontologia. }\end{array}$ \\
\hline $\begin{array}{l}\text { Crossetti, Bittencourt, } \\
\text { Schaurich, TancciniI, } \\
\text { Antunes }^{25}\end{array}$ & 2009 & $\begin{array}{l}\text { Revista } \\
\text { Gaúcha } \\
\text { Enfermagem }\end{array}$ & PCS & $\begin{array}{l}\text { Discutiram estratégias de ensino utilizadas na enfermagem para desenvolver habilidades do } \\
\text { pensamento crítico. Concluíram que o desenvolvimento de habilidades do pensamento crítico } \\
\text { pode proporcionar a formação de profissionais mais críticos e reflexivos. }\end{array}$ \\
\hline Lunney $^{23}$ & 2010 & $\begin{array}{l}\text { Journal of } \\
\text { Nursing } \\
\text { Terminologies } \\
\text { and } \\
\text { Classifications }\end{array}$ & PCS & $\begin{array}{l}\text { Descreveu a importância do pensamento crítico em enfermagem, necessário no processo de } \\
\text { diagnóstico. Afirmou que a habilidade de Pensamento Crítico possibilita melhores ações ou } \\
\text { intervenções após o diagnóstico. Afirmou que a habilidade de Pensamento Crítico possibilita } \\
\text { melhores ações ou intervenções após o diagnóstico. }\end{array}$ \\
\hline Cerullo, $\mathrm{Cruz}^{26}$ & 2010 & $\begin{array}{l}\text { Revista Latino- } \\
\text { Americana de } \\
\text { Enfermagem }\end{array}$ & PCS & $\begin{array}{l}\text { Identificaram e analisam estudos sobre raciocínio clínico e pensamento crítico em enfermagem. } \\
\text { Afirmaram que é preciso investir na formação e transformar o pensamento crítico em uma } \\
\text { unidade de conteúdo, mas desconsideram a necessidade de ser disciplina no currículo. }\end{array}$ \\
\hline Morrall, Godman ${ }^{24}$ & 2013 & $\begin{array}{l}\text { Nurse } \\
\text { Education } \\
\text { Today }\end{array}$ & PCS & $\begin{array}{l}\text { Afirmaram que o ensino do pensamento crítico nas universidades do Reino Unido tem decaído. } \\
\text { Concluíram que resgatar essa prática é essencial para promover enfermeiros competentes em } \\
\text { um mundo cada vez mais complexo e globalizado, propondo que docentes e acadêmicos se } \\
\text { engajem para restabelecer o pensamento crítico em universidades. Concluíram que resgatar } \\
\text { essa prática é essencial para promover enfermeiros competentes em um mundo cada vez mais } \\
\text { complexo e globalizado. }\end{array}$ \\
\hline
\end{tabular}


(Continuação)

\begin{tabular}{|c|c|c|c|c|}
\hline Gomes, Rego ${ }^{21}$ & 2014 & $\begin{array}{l}\text { Revista } \\
\text { Brasileira } \\
\text { Educação } \\
\text { Médica }\end{array}$ & PCS & $\begin{array}{l}\text { Discutiram possíveis contribuições advindas do pensamento de Paulo Freire para a } \\
\text { transformação das escolas médicas (medicina, enfermagem e odontologia) no Brasil. } \\
\text { Concluíram que apenas mudanças de método são insuficientes para atingir as competências } \\
\text { humanísticas preconizadas. É preciso despertar habilidades e pensamento crítico para formação } \\
\text { de profissionais críticos e reflexivos. }\end{array}$ \\
\hline $\begin{array}{l}\text { Parker S, Mayner L, } \\
\text { Michael Gillham }^{34}\end{array}$ & 2015 & $\begin{array}{l}\text { Nursing and } \\
\text { Midwifery } \\
\text { Studies }\end{array}$ & PCS & $\begin{array}{l}\text { Desenvolveram software auto orientado que fornece consistentes orientações a alunos de } \\
\text { enfermagem estruturada com desenho que possibilita desenvolver habilidades como o } \\
\text { pensamento crítico. }\end{array}$ \\
\hline $\begin{array}{l}\text { Kaddoura, Van-Dyke, } \\
\text { Yang }^{4}\end{array}$ & 2016 & $\begin{array}{l}\text { Nursing \& } \\
\text { Health } \\
\text { Sciences }\end{array}$ & PCS & $\begin{array}{l}\text { Identificaram a necessidade de implementar uma nova estratégia para ensinar aos alunos as } \\
\text { habilidades de pensar criticamente e desenvolveram uma estratégia experimental adotando o } \\
\text { mapa conceitual como instrumento para estimular o desenvolvimento do pensamento crítico. }\end{array}$ \\
\hline Yeom, Ahn, Kim ${ }^{15}$ & 2016 & Nursing Ethics & PCS & $\begin{array}{l}\text { Perceberam um subdomínio de sensibilidade moral e de curiosidade além de um subdomínio } \\
\text { da disposição do pensamento crítico com correlações positivas significativas entre } \\
\text { sensibilidade moral e pensamento crítico ao utilizar o Critical Thinking Disposition } \\
\text { Questionnaire com } 70 \text { alunos de graduação em enfermagem. }\end{array}$ \\
\hline Allen, More ${ }^{9}$ & 2004 & $\begin{array}{l}\text { Journal of } \\
\text { Dental } \\
\text { Education }\end{array}$ & & $\begin{array}{l}\text { Enfatizaram que o pensamento crítico é essencial na tomada de decisão. O conteúdo deve ser } \\
\text { interessante. Afirmaram a necessidade de reestruturar o currículo de Odonto-logia na pré- } \\
\text { clínica, que seja contextualizado e proporcione base mais forte para o ciclo clínico. Propuseram } \\
\text { currículo centrado em pacientes com perfis de necessidades de risco, doença e tratamento } \\
\text { diferenciado, no qual os alunos sejam obrigados a pensar global e criticamente, coletar dados } \\
\text { para avaliação precisa dos riscos e definir plano de tratamento. }\end{array}$ \\
\hline Araújo $^{7}$ & 2006 & $\begin{array}{l}\text { Ciência \& } \\
\text { Saúde Coletiva }\end{array}$ & $\mathrm{PCO}$ & $\begin{array}{l}\text { Questionaram o perfil dos odontólogos egressos das universidades e considera a necessidade } \\
\text { de uma revisão sobre essa formação nas faculdades de odontologia do Brasil. Afirmaram que } \\
\text { o aluno deve ser agente ativo no processo de ensino, e usar o pensamento crítico em relação às } \\
\text { ações de sua própria pratica profissional. }\end{array}$ \\
\hline $\mathrm{Katz}^{33}$ & 2006 & $\begin{array}{l}\text { Journal of } \\
\text { Evidence- } \\
\text { Based Dental } \\
\text { Practice }\end{array}$ & $\mathrm{PCO}$ & $\begin{array}{l}\text { Descreveu por meio de uma experiência na NYU, a importância do Ensino do Pensamento } \\
\text { Crítico no início da graduação em odontologia. Sugeriu que o mesmo seja integrado no } \\
\text { currículo de odontologia com um número mínimo de horas necessárias }\left(72 \mathrm{~h} \text { no } 1^{\circ} \text { ano e } 6 \mathrm{~h} \text { em }\right. \\
\text { cada ano sucessivo }=90 \mathrm{~h}) \text {, a fim de desenvolver habilidade crítica aguçada. }\end{array}$ \\
\hline
\end{tabular}


(Continuação)

\begin{tabular}{|c|c|c|c|c|}
\hline Hendricson et al. ${ }^{32}$ & 2006 & $\begin{array}{l}\text { Journal of } \\
\text { Dental } \\
\text { Education }\end{array}$ & $\mathrm{PCO}$ & $\begin{array}{l}\text { Forneceram orientação às escolas de odontologia na concepção de currículos de cursos de } \\
\text { odontologia. Descreveram possíveis melhores práticas educacionais para ajudar os estudantes } \\
\text { de odontologia adquirir a capacidade de trabalhar como um dentista generalista com visão } \\
\text { crítica e reflexiva. }\end{array}$ \\
\hline $\begin{array}{l}\text { Horenstein, Childs, } \\
\text { Graf }^{18}\end{array}$ & 2009 & $\begin{array}{l}\text { Journal of } \\
\text { Dental } \\
\text { Education }\end{array}$ & $\mathrm{PCO}$ & $\begin{array}{l}\text { Verificaram a aplicação do pensamento crítico para os docentes a fim de melhorar a prática } \\
\text { docente no ensino. Demonstraram a incorporação de conceitos de pensamento crítico em } 63 \% \\
\text { do grupo, sugerindo que a instrução do corpo docente pode resultar em melhor estímulo ao } \\
\text { pensamento crítico dos discentes. }\end{array}$ \\
\hline $\begin{array}{l}\text { Mattheos, Ucer, Van } \\
\text { De Velde, Nattestad }\end{array}$ & 2009 & $\begin{array}{l}\text { European } \\
\text { Journal of } \\
\text { Dental } \\
\text { Education }\end{array}$ & $\mathrm{PCO}$ & $\begin{array}{l}\text { Afirmaram que a aprendizagem em ambientes acadêmicos está fortemente relacionada com a } \\
\text { forma como os alunos são avaliados. Concluíram que a habilidade crítica de aprendizagem ao } \\
\text { longo da vida pessoal e profissional é construída no meio acadêmico, por meio do estímulo à } \\
\text { auto-avaliação. }\end{array}$ \\
\hline Mcharg, Kay ${ }^{11}$ & 2009 & $\begin{array}{l}\text { British Dental } \\
\text { Journal }\end{array}$ & $\mathrm{PCO}$ & $\begin{array}{l}\text { Descreveram a necessidade de envolver o aluno no aprendizado crítico, abordado em três } \\
\text { domínios: "Mão "-psicomotor (habilidades técnicas); "Coração"-o domínio afetivo (empatia e } \\
\text { comportamento) e a "Cabeça"- domínio cognitivo (o pensamento crítico), que devem ser } \\
\text { contextualizados em um, baseado em currículo integrado, onde os estudantes tratam pacientes } \\
\text { de meio a } 1 \text { ano, que os ajuda a apreciar a pertinência das 'mãos', 'coração' e 'cabeça' da } \\
\text { odontologia e com eles começam a construir seu conhecimento e experiência. }\end{array}$ \\
\hline $\begin{array}{l}\text { Horenstein, Childs, } \\
\text { Graf }^{19}\end{array}$ & 2010 & $\begin{array}{l}\text { Journal of } \\
\text { Dental } \\
\text { Education }\end{array}$ & $\mathrm{PCO}$ & $\begin{array}{l}\text { Pesquisaram doze membros do corpo docente (seis na Faculdade de Odontologia e seis na } \\
\text { Faculdade de Saúde e Performance Humana) sobre o desenvolvimento de habilidades de } \\
\text { pensamento crítico. Afirmaram que o ensino de habilidades de pensamento crítico e o } \\
\text { aprendizado adquirido é sustentado para ser transmitido. }\end{array}$ \\
\hline Costa, Araújo ${ }^{6}$ & 2011 & $\begin{array}{l}\text { Ciência \& } \\
\text { Saúde Coletiva }\end{array}$ & $\mathrm{CO}$ & $\begin{array}{l}\text { Sinalizaram para formas de enfrentar mudanças na formação de profissionais de Odontologia. } \\
\text { Afirmam que o aluno deve saber, saber-fazer, saber-ser, com visão questionadora crítica da } \\
\text { própria prática. }\end{array}$ \\
\hline Khatami, Macentee ${ }^{28}$ & 2011 & $\begin{array}{l}\text { European } \\
\text { Journal of } \\
\text { Dental } \\
\text { Education }\end{array}$ & $\mathrm{PCO}$ & $\begin{array}{l}\text { Afirmaram que a abordagem aos cuidados em Odontologia tem evoluído a partir de um foco estreito } \\
\text { sobre a doença oral a abordagem dos determinantes psicossociais da saúde bucal. Concluíram que o } \\
\text { pensamento crítico é habilidade que melhora a resolução de problemas, despertando profissionalismo } \\
\text { e competência social e cultural e auxilia nos procedimentos de maior complexidade. }\end{array}$ \\
\hline
\end{tabular}


(Continuação)

\begin{tabular}{|c|c|c|c|c|}
\hline $\begin{array}{l}\text { Johnsen, Lipp, } \\
\text { Finkelstein, } \\
\text { Cunningham-Ford }^{13}\end{array}$ & 2012 & $\begin{array}{l}\text { Journal of } \\
\text { Dental } \\
\text { Education }\end{array}$ & PCO & $\begin{array}{l}\text { Afirmaram que o profissional da saúde para exercer o cuidado centrado no paciente precisa } \\
\text { envolver domínios de conhecimento, habilidades técnicas e pensamento crítico, que se } \\
\text { desenvolve à partir de um conteúdo bem planejado. Ponderaram que a odontologia, é ensinada } \\
\text { em direção ao conhecimento e habilidades técnicas }\end{array}$ \\
\hline $\begin{array}{l}\text { Jonas-Dwyer, Abbott, } \\
\text { Boyd }^{22}\end{array}$ & 2013 & $\begin{array}{l}\text { European } \\
\text { Journal of } \\
\text { Dental } \\
\text { Education }\end{array}$ & $\mathrm{PCO}$ & $\begin{array}{l}\text { Mostraram que o pensamento crítico se tornou uma presença crescente no currículo dos cursos } \\
\text { de odontologia. Afirmaram a necessidade de desenvolver pessoal, cultural e profissionalmente, } \\
\text { os profissionais de saúde. }\end{array}$ \\
\hline $\begin{array}{l}\text { Dougall, Thompson, } \\
\text { Faulks, Ting, Nunn }\end{array}$ & 2014 & $\begin{array}{l}\text { European } \\
\text { Journal of } \\
\text { Dental } \\
\text { Education }\end{array}$ & $\mathrm{PCO}$ & $\begin{array}{l}\text { Preconizaram que é essencial desenvolver habilidades críticas e reflexivas para na prática } \\
\text { atingirem excelência profissional. Relataram a incorporação no currículo de odontologia, a } \\
\text { disciplina de Cuidados Especiais, para estimular os alunos nos conhecimentos, habilidades } \\
\text { críticas e reflexivas e possibilitar atitudes mais seguras. }\end{array}$ \\
\hline $\begin{array}{l}\text { Mitchell, Overman, } \\
\text { Forrest }^{29}\end{array}$ & 2014 & $\begin{array}{l}\text { Journal of } \\
\text { Evidence- } \\
\text { based Dental } \\
\text { practice }\end{array}$ & $\mathrm{PCO}$ & $\begin{array}{l}\text { Afirmaram que o pensamento crítico é essencial para a prática clínica de qualidade. Concluíram } \\
\text { que o pensamento crítico auxilia na tomada de decisão e seu uso é uma promessa para melhorar } \\
\text { os resultados de saúde bucal dos pacientes. }\end{array}$ \\
\hline $\begin{array}{l}\text { Gonzalez-Cabezas, } \\
\text { Anderson, Wright, } \\
\text { Fontana }^{35}\end{array}$ & 2015 & $\begin{array}{l}\text { Journal of } \\
\text { Dental } \\
\text { Education }\end{array}$ & $\mathrm{PCO}$ & $\begin{array}{l}\text { Avaliaram a colaboração de estudantes de odontologia em uma auto-avaliação de sua } \\
\text { performance como ferramenta para a construção de habilidades de pensamento crítico em salas } \\
\text { de aula e perceberam que ele pode ser útil e deve se expandir em outros cursos. }\end{array}$ \\
\hline
\end{tabular}


preendê-lo, além de facilitar o desempenho profissional diferenciado ${ }^{13}$. Autores ${ }^{4}$ identificaram a necessidade de implementar novas metodologias para ensinar aos alunos as habilidades de pensar criticamente $\mathrm{e}$ desenvolveram uma estratégia experimental adotando o mapa conceitual como instrumento para estimular o desenvolvimento do pensamento crítico.

Deve ficar claro que as competências se desenvolvem lentamente e de forma progressiva, sendo necessários recursos, informações e algo para colocar em ação. É importante entender que não existe uma única forma de praticar ações, ou um jeito correto de intervir em uma situação. Por outro lado, é fundamental para o profissional em saúde, ser despertado e ensinado, para que ao exercer o cuidado centrado no paciente, consiga dominar, além das habilidades técnicas e conhecimento, o pensamento crítico ${ }^{13}$.

Para tal, aos aspectos técnicos da profissão deve ser somado as habilidades do conhecimento e pensamento crítico, condição fundamental ao bom exercício da profissão ${ }^{10}$. Assim, é possível afirmar que os dois principais motores de conteúdos para moldar o ensino nos cursos da saúde deveriam ser, em primeiro lugar, as necessidades de saúde da população, e, por outro, os conhecimentos, habilidades e atitudes requeridas ao dentista para tratamento desses pacientes ${ }^{11,14}$. Ao utilizar o The Critical Thinking Disposition Questionnaire em um estudo com 70 alunos de graduação em enfermagem, Yeom, Ahn, Kim perceberam entre os pesquisados um subdomínio de sensibilidade moral e de curiosidade além de um subdomínio da disposição do pensamento crítico com correlações positivas significativas entre sensibilidade moral e pensamento crítico ${ }^{15}$.
A pesquisa desperta para a necessidade de repensar o processo de formação em cursos da área da saúde como Odontologia, Medicina e Enfermagem, adequando-se às mudanças que naturalmente acontecem nas diversas sociedades. Verificase que estudos envolvendo o pensamento crítico no processo de formação em saúde, são em maior número e realizados a mais tempo em relatos internacionais.

Diante de crescentes e contínuas mudanças, o processo de formação profissional em saúde no cenário nacional precisa incrementar pesquisas e práticas de forma a levar os alunos à reflexão crítica de suas ações durante o aprendizado no curso, aplicado à futura vida profissional ${ }^{16}$. As competências socioemocionais, como o pensamento crítico, são habilidades que se pode aprender, praticar e ensinar. Assim, acredita-se que para que se consiga alcançar esse propósito, a inclusão dessa competência na educação precisa ser intencional e por meio da seleção de estratégias de ensino que estimulem o desenvolvimento de atributos para além da habilidade clínica e técnica, mas também habilidades de controle de comportamento e o pensamento crítico na tomada de decisões. $\mathrm{O}$ domínio das diferentes estratégias requer diferentes formas de ensino, mas todos devem ser contextualizados em um sistema integrado para favorecer a aprendizagem. Então, a partir das situações vividas pelos educandos, a relação educador-educando é como um convite a conhecer e analisar a realidade de modo crítico ${ }^{17}$.

Contudo, torna-se fundamental, como descrito na literatura revisada, para ensinar os alunos a desenvolver competências por meio do uso de habilidades como o pensamento crítico, que os educadores reflitam sobre suas 
crenças e a maneira de ensinar. É fundamental reconhecer que ambos, alunos e professores, devem estar comprometidos em tomar decisões com base em seus atuais quadros de referência, o que permitiria o aumento na reflexão sobre as informações recebidas, propondo uma série de debates sobre as informações conflitantes, ponto este que exprime a habilidade de pensamento crítico $^{18,19}$.

É importante chamar atenção também para o fato de que aprender a ensinar os alunos a usar as habilidades de pensamento crítico exige mudanças fundamentais no planejamento do ensino ${ }^{20}$. Ao despertar para a necessidade de desenvolver as habilidades de pensamento crítico os profissionais podem melhorar a performance profissional. Portanto, é fundamental que os professores se mostrem comprometidos em despertar habilidades em seus alunos ${ }^{21,22 .}$

Embora a pesquisa tenha mostrado que a abordagem do pensamento crítico em cursos de Enfermagem não seja tão recente, é enfatizado que deve haver maior articulação entre a teoria e a prática. Assim, diante da concepção atual sobre saúde, o pensamento crítico reflete-se como habilidade fundamental nos cursos de graduação de Enfermagem, Medicina e Odontologia, alicerçando a formação universitária e a futura atuação desses profissionais, bem como suas relações com o processo de trabalho ${ }^{16}$.

Neste contexto, uma formação universitária centrada em modelo técnico e no desenvolvimento restrito de competência técnica não garante o êxito de atendimento completo em saúde. Somente refletindo-se criticamente sobre o processo de diagnóstico das enfermidades e as intervenções que solucionem os problemas é que acontecerão as melhores ações naquela situação ${ }^{23}$.
As mudanças de concepção de saúde e doença, assim como a relação profissional versus usuário do sistema de saúde, requerem uma preparação diferenciada desse profissional para atuar eticamente no processo de saúde-doença, realidade um pouco distante do perfil de muitos egressos atuais. Novas metodologias engajadas em pensamento crítico, que deem conta de tantas mudanças $^{24,25}$ - podem ser adotadas aumentando a capacidade de transformação que esse conceito pode gerar ${ }^{26}$.

A Lei de Diretrizes e Bases da Educação Nacional (LDB) ${ }^{27}$ foi promulgada em 1996 e propôs a elaboração das DCN para orientar a formação profissional em diferentes áreas. Em 2002, o ensino de Odontologia foi contemplado com novas DCN. Tal proposta visou superar desafios da formação do cirurgião-dentista caracterizados pelo foco em especializações, fragmentação e interesses econômicos. Segundo as novas diretrizes, o perfil do odontólogo egresso abrange um profissional com formação generalista, humanista, crítica e reflexiva, que o permita atuar em todos os níveis de atenção à saúde, apoiados no rigor técnico e científico ${ }^{10}$.

As mudanças requeridas com a implementação das DCN para o curso de Odontologia parecem complexas e ainda se percebem lacunas a serem preenchidas para que a prática odontológica generalista, crítica e reflexiva impacte na construção de ações práticas mais comprometidas com a promoção de saúde. A falta de articulação da teoria com a prática, com predomínio de uma visão descontextualizada da realidade, pode refletir um profissional com menor habilidade crítica na tomada de decisões durante sua atuação no trabalho ${ }^{21}$.

O questionamento e o diálogo são importantes na formação do profissional 
crítico e reflexivo ${ }^{7,28}$. O preparo do futuro profissional deve estar diretamente associado aos possíveis campos de trabalho para sua atuação, ao perfil profissional e às necessidades da população, numa articulação dos currículos, dos conteúdos e das necessidades. Observa-se, de fato, que ainda predominam ações pontuais, descontextualizadas dos temas emergentes, frequentemente descoladas de uma proposta pedagógica, sem questionar as necessidades do momento, apenas realimentando uma visão simplista, assistencialista e reducionista ${ }^{29}$.

Diante da literatura pesquisada e das evidentes complexidades que permeiam o contexto acadêmico de cursos de saúde, é possível afirmar que para ser cirurgiãodentista é preciso mais do que o sonho, o desejo e a destreza. Independente do contexto de sua prática, hoje o profissional de Odontologia precisa pensar criticamente, não só pelo aumento de seu capital cultural, mas também para diminuir a distância entre o avançar da ciência e o usufruir dos resultados pela população, quer seja no Brasil ou qualquer parte do mundo.

Despertou-nos o fato de que muitas dificuldades e obstáculos encontrados ao longo da atuação profissional de egressos dos cursos da área de saúde podem ter sido reflexos de uma série de desafios educacionais complexos, susceptíveis de ter impacto sobre o processo de socialização da academia em geral. Em conjunto, esses fatores têm o potencial de atuar como obstáculos, tanto de uma perspectiva de ensino de cursos da área da saúde, bem como na aprendizagem dos futuros profissionais, seu profissionalismo e competência social, auxiliando-os a lidar com as diversidades culturais e a complexidade das questões relacionadas à saúde bucal, além das constantes mudanças subjacentes à prática da odontologia $^{18,26,28,30,31}$.

Embora seja reconhecida a importância do pensamento crítico nas práticas de diagnóstico e estratégias de ação, a literatura estudada mostra que barreiras e facilitadores dessa habilidade também devem ser explorados. Tendências atuais na educação destacam a relevância de se preparar o aluno para a definição e solução de problemas, produzindo conhecimento por meio de práticas que envolvam o pensamento crítico, criativo e reflexivo, paralelamente ao cultivo de um conjunto de traços de personalidade como persistência, autoconfiança e independência de pensamento, indispensáveis a uma melhor expressão do potencial dos futuros profissionais.

A harmonia no ambiente de aprendizagem, a atmosfera, autoestima e estado de ansiedade dos alunos podem afetar a valorização do exercício do pensamento crítico pelos alunos. Os ambientes de aprendizagem devem ser seguros e livres para expressão e discussão de pensamentos. A relação de trabalho não deve ser ameaçadora, e sim, encorajadora ${ }^{14}$. Além disso, o fato de se sentir aceito e ter boa relação interpessoal com a equipe durante as atividades clínicas tem influência positiva no estímulo do pensamento crítico dos alunos ${ }^{19,23}$.

A falta de sensibilidade cultural e um sistema de ensino tradicional dificultam a disseminação e o crescimento do pensamento crítico no meio profissional e acadêmico ${ }^{20,23}$. Ao integrar um novo conceito, a conveniência cultural deve ser considerada. A ênfase na aprendizagem mecânica, o modo de ensino na sala de aula e a relação de poder entre professores e alunos desencorajam estudantes a cultivar muitas habilidades, entre elas o pensamento crítico. Apesar do cenário desfavorável de uma sociedade de consumo, 
que reconhece o técnico que produz mais e não o médico que assiste com mais qualidade seus pacientes, é necessário que se leve a sério a necessidade de se desenvolver uma teoria da democracia que ajude a atenuar, senão eliminar completamente, as diferenças e desigualdades sociais.

A literatura mostrou que é necessário dar ênfase às relações entre as disciplinas e o curso como um todo, enfatizando a posição de importância de professores, tutores e preceptores no estímulo à síntese de conhecimento das diversas disciplinas abordadas durante o curso, para a formação de um profissional generalista, que busca a visão holística do $\operatorname{ser}^{30}$. Sob olhar atento, faz-se necessário construir algumas análises que mostram que as diferentes práticas presentes no nosso cotidiano, estão interligadas no mundo contemporâneo de modo a construir um mosaico no qual o sujeito se localiza. Mostra também, que pensar criticamente, em sua polissemia, tem uma relação complexa que aponta possíveis origens e efeitos: de um lado, ela caracteriza uma série de fenômenos; por outro, mostra a fragilidade humana derivada destes, que ao mesmo tempo que os mantém, também busca alternativas para (sobre)viver neste contexto ${ }^{31}$.

Dessa forma, não basta estar com o outro, mas sim, "ser alguém para o outro". A expressão "ser-para" é muito importante porque nos mostra que estar ao lado do outro não significa necessariamente um ato moral. O "ser-para" confere uma atitude diferenciada pois aponta para a condição de "viver-com-ooutro", dividindo alegrias, tristezas, conquistas, dificuldades, atravessadas pela situação de negociação e diálogo ${ }^{31}$.

Na educação odontológica crítica, para ser pertinente, como verificado nos artigos revisados, o conhecimento não deriva de saberes desunidos, mas da orientação de conteúdos que transcendem a execução técnica dos procedimentos ${ }^{32,33}$. Indo além da técnica, o estímulo ao pensamento crítico é favorecido por estratégias de simulações, estudos de caso e entrevistas motivacionais, que podem despertar nos acadêmicos e profissionais a vontade de reexaminar as crenças e suposições em busca de evidências clínicas e científicas para apoiá-las 9,12,21,25,29.

Isso é determinado, pelo fato de que os alunos irão viver e atuar num mundo onde cada vez mais é preciso usar capacidades de pensamento crítico e exercer a autocrítica para resolver problemas e tomar decisões, não somente envolvendo como fazer intervenções operacionais, mas também saber quando intervir ou não com envolvimento éticosocial. Muito mais que dentistas qualificados científica e teoricamente, é preciso formar cidadãos com reflexão crítica, cientes da atual situação de saúde e do seu papel diante da sociedade brasileira.

Diante do cenário atual em que vivemos, de rápidas transformações e grandes desafios, é inquestionável a necessidade de instrumentalizar o aluno a prever problemas, romper barreiras, reformular conteúdos e desenvolver formas de investigação mais produtivas. Para isso, é necessário que ele esteja inserido em um ambiente que valorize e encoraje a criatividade. Por contraste, este estudo revelou novas sugestões para que, a partir dos métodos de ensino descritos anteriormente, os cursos da saúde lutem arduamente para integrar o pensamento crítico e melhorar as competências dos alunos na sua vida profissional e pessoal.

O trabalho introduz viés de linguagem, pois artigos sobre o assunto pesquisado podem buscados em outros idiomas nas bases de dados pesquisadas, contudo, não 
desmerece os resultados levantados.

\section{CONSIDERAÇÕES FINAIS}

Nos cursos superiores em saúde, e na odontologia em particular, vem ocorrendo uma implementação gradual de novos modelos educacionais, com destaque para mudanças curriculares e o uso de novas abordagens metodológicas que estimulem o desenvolvimento outras habilidades além do conhecimento técnico-científico.

Embora habilidades acadêmicas como o pensamento crítico sejam conceituadas como uma parte vital no ensino universitário em saúde, muitos estudantes partem para o exercício profissional sem ter conhecido a necessidade de desenvolver e dominar esta habilidade de maneira eficaz.

Considerando que os significados de nossas práticas dependem das convicções formuladas sobre $\mathrm{o}$ processo educativo recebido e das competências construídas, torna-se importante avançar nas produções científicas sobre o pensamento crítico na odontologia, pois a pesquisa realizada demonstrou que são escassos os estudos nacionais sobre esse tema.

Pensar criticamente pode ampliar a possibilidade de construir um novo modo de praticar a atenção à saúde, mas para isso é preciso refletir sobre o atual perfil de trabalho e de trabalhadores. A formação e a qualificação dos profissionais da saúde devem ser orientadas pelas necessidades da população e auxiliadas pelo questionamento da realidade vivida.

Objetivando-se que às técnicas e teorias ensinadas seja somado, e não substituído, o pensamento crítico dos alunos, diante de situações práticas em saúde, explorar o estímulo ao pensamento crítico pode proporcionar mudanças nas práticas profissionais que tenham impacto direto na assistência e cuidado em saúde geral e saúde bucal. Diante do fato do pensamento crítico ser um conceito em desenvolvimento na área de saúde, tanto na Medicina, como na Enfermagem e na Odontologia, o estudo pode colaborar para despertar para necessidade de desenvolver novas pesquisas sobre o assunto.

\section{ABSTRACT \\ Critical thinking and professional training in Dentistry: a narrative literature review}

The study aimed to investigate the literature and to describe the importance of critical thinking-based learning in healthcare, mainly in Dental professional training. The study deals with a literature review on the training of health professionals. Data were collected from Medline, Lilacs and SciELO. Full texts were collected from journals in the area of health sciences, specifically Medicine, Nursing and Dentistry. The descriptive analysis conducted showed 3 categories which oriented the study. The final analysis included 25 of the 211 papers retrieved. The results showed that $76.2 \%$ of them were published between 2009 and 2015, only $33.3 \%$ are from Brazil and $66.7 \%$ expressed analysis and results of international publications. It was also found that $66.7 \%$ studies addressed critical thinking in Dentistry, but only $9.5 \%$ were conducted in Brazil. In conclusion, the skills involving critical thinking bring evident benefits in training health professionals, resulting in more reflexive ant questioning individuals.

Descriptors: Competency-Based Education. Dentistry. Higher Education. Thinking. Review.

\section{REFERÊNCIAS}

1. Alfaro-Lefevre R. Pensamento crítico em enfermagem: um enfoque prático. Artes Médicas. 1996.

2. Sordi MRL, Bagnato MHS. Subsídios para uma formação profissional crítico- 
reflexiva na área de saúde: o desafio da virada do século. Rev Latino-Am Enferm. 1998;6(2):83-8.

3. Gonçalves CMB, Siqueira LMC. A docência na educação superior: saberes e identidades. Grupo de Trabalho Didática. Disponível em: http://www.uftm.edu.br/ upload/ensino/Artigo_TCC_Lizarda.pdf Acesso em: 12 de fevereiro de 2016.

4. Kaddoura M, Van-Dyke, O, Yang, Q. Impact of a concept map teaching approach on nursing students' critical thinking skills. Nurs Health Sci. 2016;18. Disponível em: http://onlinelibrary. wiley.com/doi/10.1111/nhs.12277/abstra ct;jsessionid=D2799303AA63100A6CC 76DA736954F95.f01t03 Acesso em: 12 de fevereiro de 2016.

5. Rainbolt G. Pensamento crítico. Rev Pesq Filos: Fundamento. 2010;1(1):35-50.

6. Costa ICC, Araújo MNT. Definição do perfil de competências em saúde coletiva a partir da experiência de cirurgiõesdentistas atuantes no serviço público. Ciênc Saúde Colet. 2011;16(1):1181-9.

7. Araújo ME. Palavras e silêncio na educação superior em odontologia. Ciênc Saúde Colet. 2006;1(11):179-82.

8. Andreou C, Papastavrou E, Merkouris A. Learning styles and critical thinking relationship in baccalaureate nursing education: a systematic review. Nurse Educ Today. 2014; 34:362-71.

9. Allen KL, More FG. Clinical simulation and foundation skills: an integrated multidisciplinary approach to teaching. J Dent Educ. 2004; 68(4):468-74.

10. Conselho Nacional de Educação. Parecer CNE No 009/01. Diretrizes Curriculares Nacionais para a Formação de Professores da Educação Básica, em cursos de licenciatura, de graduação plena. Brasília, DF; DOU; 2002.

11. Mcharg J, Kay EJ. Designing a dental curriculum for the twenty-first century. Br Dent J. 2009; 207(10):493-7.

12. Miranda KCL, Barroso MGT. A contribuição de Paulo Freire à prática e educação crítica em Enfermagem. Rev Lat-Am Enf. 2004;12(4):631-5.

13. Johnsen DC, Lipp MJ, Finkelstein MW, Cunningham-Ford MA. Guiding dental student learning and assessing performance in critical thinking with analysis of emerging strategies. J Dent Educ. 2012; 76(12):1548-58.

14. Mattheos N, Ucer C, Van De Velde T, Nattestad A. Assessment of knowledge and competencies related to implant dentistry in undergraduate and postgraduate university education. Eur $\mathbf{J}$ Dent Educ. 2009; 13:55-65.

15. Yeom, HA, Ahn, SH, Kim, SJ. Effects of ethics education on moral sensitivity of nursing students. Nurs Ethics. 2016; 24. Disponível em: http://nej.sagepub.com/ content/early/2016/01/21/096973301562 2060.abstract Acesso em: 14 de fevereiro de 2016.

16. Fagundes NC, Burnham TF. Discutindo a relação entre espaço e aprendizagem na formação de profissionais de saúde. Comunic Saúde Educ. 2005; 9(16):10514.

17. Freire P. Pedagogia do oprimido. São Paulo: Paz e Terra. 43. ed. 2005.

18. Horenstein, LSB, Childsl GS, Graf R. Promoting the teaching of critical thinking skills through faculty development. J Dent Educ. 2009; 73(6):665-75.

19. Horenstein, LSB, Childs GS, Graf R. Observation and assessment of faculty development learning outcomes. J Dent Educ. 2010; 74(11):1245-54.

20. Pires ROM, Bueno SMV. Freire e formação para o Sistema Único de Saúde: o enfermeiro, o médico e o odontólogo. Acta Paul Enferm. 2009; 22(4):439-44.

21. Gomes AP, Sergio R. Paulo Freire: contribuindo para pensar mudanças de estratégias no ensino de medicina. Rev Bras Educ Med. 2014; 38(3):299-313.

22. Jonas-Dwyer DRD, Abbott PV, Boyd N. First reflections: third-year dentistry students' introduction to reflective 
practice. Eur J Dent Educ. 2013;17(1): 64-9.

23. Lunney M. Use of critical thinking in the diagnostic process. Int $\mathbf{J}$ Nurs Terminol Classif. 2010;21(2):82-8.

24. Morrall P, Goodman B. Critical thinking, nurse education and universities: Some thoughts on current issues and implications for nursing practice. Nurse Educ Today. 2013;33(9):935-7.

25. Crossetti MGO, Bittencourt GKGD, Schaurich D, TancciniI T, Antunes M. Estratégias de ensino das habilidades do pensamento crítico na enfermagem. Rev Gaúcha Enferm. 2009;30(4):732-41.

26. Cerullo JASB, Cruz DALM. Raciocínio clínico e pensamento crítico. Rev LatinoAm Enf. 2010;18(1):124-9.

27. Lei 9.394, de 20 de dezembro de 1996. Estabelece as Diretrizes e Bases da Educação Nacional. Diário Oficial da União. Brasília, DF, 1996.

28. Khatami S, Macentee M. Evolution of clinical reasoning in dental education. $\mathrm{J}$ Dent Educ. 2011;75(3):321-8.

29. Mitchell SH, Overman P, Forrest J L. Critical thinking in patient centered care. J Evid Bas Dent Pract. 2014;14:235-9.

30. Dougall A, Thompson SA, Faulks D, Ting G, Nunn J. Guidance for the core content of a Curriculum in Special Care Dentistry at the undergraduate level. Eur J Dent Educ. 2014;18(1):39-45.

31. Bauman Z. Capitalismo Parasitário. Rio de Janeiro: Zahar, 2010.

32. Hendricson WD, Andrieu SC, Chadwick DG, Chmar JE, Cole JR, George MC, et al. Educational strategies associated with development of problem-solving, critical thinking, and self-directed learning. J Dent Educ. 2006;70(9): 925-36.

33. Katz RV. The importance of teaching critical thinking early in Dental education: concept, flow and history of the NYU 4-Year Curriculum or Miracle on 24th Street: the EBD. J Evid Base Dent Pract. 2006;6:62-71.
34. Parker S, Mayner L, Michael Gillham D. E-learning for critical thinking: using nominal focus group method to inform software content and design. Nurs Midwifery Stud. 2015; 4 (4). Disponível em: http://www.ncbi.nlm.nih.gov/pmc/ articles/PMC4733504/pdf/nms-0430471. pdf Acesso em: 14 de fevereiro de 2016.

35. Gonzalez-Cabezas C, Anderson OS, Wright MC, Fontana M. Association between Dental student-developed exam questions and learning at higher cognitive levels. J Dent Educ. 2015; 79(11):1295304.

Correspondência para:

Cynthia Moura Louzada Farias

e-mail: cynthialousada1@ hotmail.com

Av. Marechal Campos, 1468

Maruipe 29043-900 Vitória/ES 